\title{
Research Paper: Coenzyme Q10 Insufficiency Contributes to the Duration and Frequency of Seizures in Epileptic Patients
}

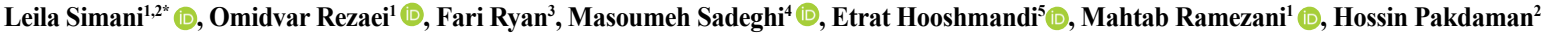

1. Skull Base Research Center, Loghman Hakim Hospital, Shahid Beheshti University of Medical Sciences, Tehran, Iran.

2. Brain Mapping Research Center, Shahid Beheshti University of Medical Sciences, Tehran, Iran.

3. Centre for Research in Neuroscience, The Research Institute of the McGill University Health Center, Montreal, Quebec, Canada.

4. Department of Epidemiology, School of Public Health and Safety, Shahid Beheshti University of Medical Sciences, Tehran, Iran.

5. Clinical Neurology Research Center, Shiraz University of Medical Sciences, Shiraz, Iran.

\begin{tabular}{|c|c|}
\hline $\begin{array}{l}\text { Use vour device to scan } \\
\text { and read the article online }\end{array}$ & Citation: Simani, L., Rezaei, O., Ryan, F., Sadeghi, M., Hooshmandi, F., \& Ramezani, M., et al. (2020). Coenzyme Q10 Insuf- \\
\hline 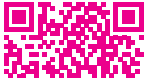 & $\begin{array}{l}\text { ficiency Contributes to the Duration and Frequency of Seizures in Epileptic Patients. Basic and Clinical Neuroscience, 11(6), } \\
765-772 . \text { http://dx.doi.org/10.32598/bcn.11.6.1100.1 }\end{array}$ \\
\hline 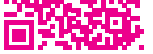 & doij ${ }^{\circ}$ http://dx.doi.org/10.32598/ben.11.6.1100.1 \\
\hline
\end{tabular}

\section{(1) (3)}

Article info:

Received: 27 Oct 2018

First Revision: 20 Nov 2018

Accepted: 23 Apr 2019

Available Online: 01 Nov 2020

Keywords:

Coenzyme Q10, Epileptic seizures, Oxidative stress, Clinical signs of seizure

\begin{abstract}
A B S T RA C T
Introduction: Oxidative stress has recently emerged as a possible mechanism in the pathogenesis of epilepsy. Coenzyme Q10 (CoQ10) is a strong endogenous antioxidant that protects cells from lipid oxidation and Reactive Oxygen Species (ROS) production; however, the impact of CoQ10 on seizure characteristics in epileptic patients is unclear.

Methods: The current study enrolled patients with Epileptic Seizure (ES) to evaluate their serum concentration of CoQ10 and to investigate whether a relationship exists between CoQ10 levels with the duration, frequency, and type of seizure.

Results: A total of 39 patients with epileptic seizures and 35 healthy controls were included in the study. The levels of CoQ10 in ES patients were significantly lower in comparison with healthy controls $(11.99 \pm 5.93 \mathrm{vs}(\mathrm{ng} / \mathrm{ml}) .16 .48 \pm 4.20(\mathrm{ng} / \mathrm{ml}) \mathrm{P}<0.001)$. We also found that the duration of epilepsy and seizure frequency was negatively correlated with serum CoQ10 levels.

Conclusion: These findings indicate that CoQ10 deficiency might substantially contribute to the clinical signs of epileptic patients.
\end{abstract}




\section{Highlights}

- CoQ10 deficiency plays a role in the pathogenesis of Epileptic Seizure (ES).

- Decrement of CoQ10 level in ES may be contributed to an increase in the frequency and duration of the seizure.

- There is no association between the type of seizure and CoQ10 levels in epileptic patients.

\section{Plain Language Summary}

Decreasing antioxidants plays an important role in the pathogenesis of epilepsy. Coenzyme Q10 (CoQ10) is a strong endogenous antioxidant that protects the body against several diseases; however, the impact of CoQ10 in epilepsy is unclear. We evaluated the serum concentration of CoQ10 to test if there is any relationship between CoQ10 levels and clinical manifestation of seizure. We found that the levels of CoQ10 in epileptic patients were significantly lower compared to healthy volunteers. We also found that the clinical manifestation of the seizure (duration of epilepsy and seizure frequency) were negatively correlated with serum CoQ10 levels.

\section{Introduction}

$\mathbf{P}$

erturbation in the antioxidants and oxidants equilibrium results in a phenomenon called oxidative stress which is recently suggested as a contributing mechanism to epilepsy (Sudha, Rao, \& Rao, 2001). The association between the generation of free radicals and their scavengers by antioxidant systems has been established in epilepsy. An increase in the formation of reactive oxygen species (ROS) has been viewed in an animal model of epilepsy as well as in neurodegeneration observed in epileptic humans (Militão, Ferreira, \& de Freitas, 2010). Recent evidence shows that pro-oxidant/antioxidant imbalance can also increase the probability of seizure recurrence (Ercegovac et al., 2013). Besides, elevation in ROS production may prolong seizures duration and the development of subsequent epilepsy (Martinc, Grabnar, \& Vovk, 2012; Méndez-Armenta, Nava-Ruíz, Juárez-Rebollar, Rodríguez-Martínez, \& Yescas Gómez, 2014).

Coenzyme Q10 (known as coenzyme Q, ubidecarenone, and ubiquinone) is a lipophilic compound mainly located in mitochondrial membranes of all tissue cells and acts as a crucial enzyme cofactor in the mitochondrial respiratory chain for the synthesis of Adenosine Triphosphate (ATP) (Matthews, Yang, Browne, Baik, \& Beal, 1998). It also plays an important role as a potent antioxidant either directly by scavenging the free radicals or indirectly by regenerating other antioxidants, such as vitamin E (Matthews et al., 1998; Sattarinezhad, Shafaroodi, Sheikhnouri, Mousavi, \& Moezi, 2014).
Since disturbance in the activity of antioxidative defense mechanisms may be accounted for the pathogenesis of epilepsy, and CoQ10 functions as a strong endogenous antioxidant, it is imperative to unravel the effects of CoQ10 in epileptic patients. While in vivo studies have shown the detrimental effect of CoQ10 insufficiency in epilepsy (de Sales Santos et al., 2010; Tawfik, 2011), no human studies have addressed this issue. Therefore, the first aim of the present study was to quantify the serum concentration of CoQ10, and the second aim was to figure out the clinical relevance of the type, frequency, and duration of seizures with CoQ10 levels.

\section{Methods}

\subsection{Study population}

This analytical cross-sectional study was performed in the Long Term Monitoring (LTM) Ward of the Loghman Hakim Hospital, Tehran, Iran. Medical Ethics Committee of Shahid Beheshti University of Medical Sciences approved the study (Ethics Committee number: IR.SBMU. RETECH.REC.1396.411). All subjects agreed and completed the written informed consent.

The case group included patients who were admitted to the LTM ward of Loghman Hakim Hospital due to repeated epileptic seizures. Their demographic and seizure characteristics such as type, frequency, duration, and repetition as well as the history of epileptic medications were collected through an interview with the patients. All patients underwent at least 48 hours of continuous scalp EEG monitoring to capture enough habitual seizures. The epilepsy type was confirmed by an epileptologist based 
on ictal and interictal EEG findings and the semiology of the seizures. The patients with any other medical, neurological, psychiatric disorders, and history of recent head trauma were excluded from the study. The control group consisted of age and gender-matched healthy volunteers with no history of seizure. Either patients or controls were not included in the study if they received antioxidant medications such as vitamin $\mathrm{C}$, corticosteroids, and nonsteroidal anti-inflammatory drugs within the past month.

\subsection{Blood collection and measurement of Coen- zyme Q10 levels}

Venous blood samples were taken from all patients within 24 hours of a habitual seizure. Five milliliters of venous blood was collected from each study participant and centrifuged at $4000 \times \mathrm{g}$ for 10 minutes $\left(\right.$ at $4^{\circ} \mathrm{C}$ ). Then serum was immediately separated and aliquots were stored at $-80^{\circ} \mathrm{C}$.

Serum CoQ10 levels were measured using the commercially available ELISA (enzyme-linked immunosorbent assay) kit, according to the manufacturer's instructions (ZB-13164S-H9648; ZellBio GmbH, Ulm, Germany). The serum CoQ10 levels were expressed as $\mathrm{ng} / \mathrm{mL}$. The intra-assay and inter-assay coefficients of variation were $10 \%$ and $12 \%$, respectively.

\subsection{Statistical analysis}

We used the mean difference and $95 \%$ confidence interval for the absolute difference of CoQ10 level $(\mathrm{ng} / \mathrm{mL})$ in the study groups. To support the normality of distribution, the Kolmogorov-Smirnov test was used. Continues and categorical data were reported by mean \pm standard deviation and number (percentage), respectively. The independent sample t-test and Chi-square tests were applied to compare quantitative and categorical variables between the study groups. One-way Analysis of Variance (ANOVA) was used to assess the level of CoQ10 in different types of epilepsy. Furthermore, multiple linear regression analysis was performed to explore the association between $\mathrm{CoQ} 10$ and epilepsy characteristics (duration, frequency, and type of seizure). Statistical analysis was performed using SSPS version 16.0 (SPSS, Inc., Chicago, IL, USA). The statistical significance level was considered as $\mathrm{P}<0.05$.

\section{Results}

Demographic and clinical data for the study subjects are presented in Table 1. A total of 39 patients with Epileptic Seizures (ES), 22 men and 17 women with a mean age of $29.79 \pm 11.89$ years were included in the study. The control group consisted of 35 healthy volunteers, 13 men and 22 women with a mean age of $26.97 \pm 6.62$ years. There was no significant difference between the age and gender of the two groups.

Table 1. Demographic and clinical characteristics of subjects in the study groups

\begin{tabular}{|c|c|c|c|c|}
\hline \multirow{2}{*}{\multicolumn{2}{|c|}{ Variables }} & \multicolumn{2}{|c|}{ No. (\%)/ Mean \pm SD } & \multirow{2}{*}{$\mathbf{P}$} \\
\hline & & Case Group ( $n=39$ ) & Comparison Group ( $n=35$ ) & \\
\hline \multirow{3}{*}{ Sex } & Male & $22(56.4)$ & $13(43.6)$ & \multirow{3}{*}{0.11} \\
\hline & & & & \\
\hline & Female & $17(37.1)$ & $22(62.9)$ & \\
\hline \multicolumn{2}{|c|}{ Age } & $29.7 \pm 11.89$ & $26.97 \pm 6.62$ & 0.22 \\
\hline \multicolumn{2}{|c|}{ Epilepsy duration (years) } & $13.51 \pm 11.44$ & $N A^{*}$ & - \\
\hline \multicolumn{2}{|c|}{ Seizure frequency (month) } & $11.92 \pm 21.21$ & NA & - \\
\hline \multirow{2}{*}{ Type of epilepsy } & Focal onset & $28(71.8)$ & NA & - \\
\hline & Generalized onset & $11(28.2)$ & NA & - \\
\hline \multirow{3}{*}{ Anti-epileptic drugs used } & Monotherapy & $9(23.1)$ & NA & - \\
\hline & Polytherapy & 30 (76.9) & NA & - \\
\hline & Serum CoQ10 (ng/ml) & $11.99 \pm 5.93$ & $16.48 \pm 4.20$ & $<0.001$ \\
\hline
\end{tabular}

*Not applicable. 
Table 2. Association between serum level of CoQ10 and demographic characteristics of study subjects in multiple linear regression model $\left(R^{2}=0.42\right)$

\begin{tabular}{|c|c|c|c|c|c|c|}
\hline & \multirow{2}{*}{ Variables } & \multirow{2}{*}{$\begin{array}{c}\text { Mean } \pm \text { SD } \\
\text { Serum Level of CoQ10 }\end{array}$} & \multirow{2}{*}{$\begin{array}{c}\text { Crude } \\
\beta \text { Coefficient }\end{array}$} & \multicolumn{3}{|c|}{ Adjusted } \\
\hline & & & & $\mathbf{P}$ & $\beta$ Coefficient & $\mathbf{P}$ \\
\hline Age & - & - & -0.11 & 0.09 & -0.079 & 0.210 \\
\hline Sex & $\begin{array}{c}\text { Male (reference) } \\
\text { Female }\end{array}$ & $\begin{array}{c}13.60 \pm 5.73 \\
14.57 \pm 5.5\end{array}$ & 0.97 & 0.46 & 0.114 & 0.927 \\
\hline Group & $\begin{array}{c}\text { Epileptic seizure(reference) } \\
\text { Healthy }\end{array}$ & $\begin{array}{l}11.99 \pm 5.93 \\
16.48 \pm 4.20\end{array}$ & 4.49 & $<0.001$ & 4.243 & 0.001 \\
\hline
\end{tabular}

NEUR SCIENCE

Table 1 presents the serum concentration of CoQ10 in ES patients compared to healthy subjects. Our finding revealed that the ES patients had a significantly lower level of CoQ10 compared to the control group $(\mathrm{P}<0.001)$.

The association of the study group, age, and sex with a serum level of CoQ10 was examined. There was a statistically significant positive association between the study group and level of CoQ10 in both crud ( $\beta$ : 4.49, $\mathrm{P}<0.001)$ and adjusted $(\beta: 4.24, \mathrm{P}<0.001)$ models. As shown in Table 2, the healthy group showed a $4.24 \mathrm{ng} /$ $\mathrm{mL}$ increase in CoQ10 concentration when they were adjusted for age and sex covariates. It means that the observed positive significant association between study groups and serum level of CoQ10 was not attenuated after adjusting for age and sex of study subjects (Table 2).

We also measured the association of seizure type (generalized seizures versus focal seizures) with serum concentration of CoQ10. Among the 39 recorded ES episodes, 28 attacks (71.8\%) were focal seizures and 11 attacks $(28.2 \%)$ were generalized convulsive seizures. No statistically significant correlation was found between the type of seizure and serum CoQ10 level.

Regarding the clinical features of epilepsy, we assessed the relationship between serum levels of CoQ10 and the frequency and duration of seizures using the Pearson correlation test. The mean seizure frequency was $11.92 \pm 21.21$ attacks per month, and the epilepsy duration was $13.51 \pm 11.44$ years. Interestingly, our results showed a negative significant correlation between duration of epilepsy and serum levels of CoQ10 $(r=-0.37, P$ $=0.02$ ). A similar result was also detected when the correlation was examined between seizure frequency and levels of CoQ10 $(\mathrm{r}=-0.36, \mathrm{P}=0.02)$.

\section{Discussion}

Oxidative stress triggered by diminished endogenous antioxidants or enhanced oxidants formation is currently considered as a possible mechanism underlying epileptic seizures (Martinc, Grabnar, \& Vovk, 2014). CoQ10 is a strong antioxidant that protects cells from oxidative damage through inhibiting certain enzymes involved in the formation of ROS and generating other antioxidants (El-ghoroury et al., 2009; Bhardwaj, \& Kumar, 2016). However, the impact of CoQ10 on seizure characteristics in ES patients is not clear. In this study, we provide novel insights into the role of CoQ10 insufficiency in the pathophysiology of epileptic seizures. We demonstrate, for the first time, that serum concentration of CoQ10 decreases in ES patients. Our findings show that increasing the deficiency of CoQ10 results in more frequent and longer-lasting epilepsy.

It seems that the endogenous antioxidants and their repair capacity, which normally overcome the increased production of oxidants in cells, is reduced in ES patients. We thus assessed the serum levels of CoQ10, as an endogenous antioxidant, in these patients. Although baseline levels of CoQ10 could not be measured in the ES patients in our study, we found that their serum concentrations of CoQ10 were significantly lower than the control group. Decreased CoQ10 levels have also been shown to associate with various neurological diseases, including cerebral infarction, neurodegenerative processes, cerebral ataxia, and many other brain disorders (Lamperti et al., 2003, Spindler, Beal, \& Henchcliffe, 2009; Ramezani, Sahraei, Simani, Heydari, \& Shahidi, 2020; Simani, et al., 2018). CoQ10 deficiency could result in the enhancement of electrons transport to oxygen, which leads to the significant generation of superoxide anion $\left(\mathrm{O}_{2}\right)$ in mitochondria (Chew, \& Watts, 2004). It 
then may damage the cellular components due to the elevation of ROS and reduction in ATP production; however, the effect of insufficient CoQ10 in the former is more prominent in the pathogenesis of the diseases (Lalkovičová and Danielisová 2016; Milanlioglu et al., 2016). On the other hand, an increase in free radicals and a decrease in antioxidant level has been observed in the development of epilepsy (Cengiz, Yüksel, \& Seven, 2000; Verrotti et al., 2002). Therefore, it is unclear what might underlie the decline of antioxidants such as serum CoQ10. Taken together, it can be postulated that an attenuated level of CoQ10 leads to a significant rise in ROS production, resulting in oxidative stress and thus neuronal damage in an epileptic brain.

To determine the potential role of CoQ10 in the type of seizures, we measured the correlation between these two variables. Based on our data, CoQ10 levels were not different in seizure types. This suggests that the type of epilepsy is unlikely to affect the serum concentration of CoQ10 in different ways. This finding is consistent with prior work showing no correlation between the activity of antioxidant enzymes or oxidative stress and types of seizures (Yiş, Seçkin, Kurul, Kuralay, \& Dirik, 2009).

This study provides clear evidence on a correlation between serum levels of CoQ10 and seizure frequency and duration of epilepsy. This issue may be related to free radical-mediated damage or a decrease in the activity of antioxidants caused by the deficiency of CoQ10 in ES patients (Cengiz et al., 2000; Verrotti et al., 2002). It has been shown that the risk of seizure recurrence increases with CoQ10 deficiency (Yiş et al., 2009). An absolute or relative deficiency in CoQ10 results in a remarkable increase in the transfer of electrons to molecular oxygen in mitochondria, leading to the overproduction of superoxide free radicals (Chew \& Watts, 2004). The detrimental role of free radicals in seizures has been reported in rodent studies showing that antioxidants can reduce the oxidative stress markers in epileptic animals, accompanied by the decrease of their seizure manifestations (Tan, Manchester, Reiter, Kim, \& El-Sokkary,1998; Gupta, Briyal, \& Chaudhary, 2002; Mohanan, \& Yamamoto 2002; Barros et al., 2007; Xavier et al., 2007).

Furthermore, the activity of CoQ10, as an antioxidant scavenger, inhibits lipid peroxidation (Mancuso, Orsucci, Volpi, Calsolaro, \& Siciliano, 2010). In this regard, findings from animal seizure models show that treatment of epileptic rats with CoQ10 exerts the neuroprotective effects by the removal of free radicals and reduction in lipid peroxidation levels and nitrite content, leading to ameliorate seizure severity (de Sales Santos et al., 2010; Tawfik, 2011). It has also been demonstrated that pretreatment with CoQ10 during the acute phase of pilocarpine-induced seizures results in lipid peroxidation reduction and antioxidant factors elevation, leading to an overall decrease in oxidative stress (Santos et al., 2009).

Therefore, based on our results, the negative association between seizure frequency and duration of epilepsy with CoQ10 may be related to free radical-mediated damage triggered by a deficiency in CoQ10.

In conclusion, our study demonstrated significantly decreased levels of CoQ10 in ES patients. We also found that deficiency of CoQ10 would dramatically exacerbate the clinical features of epilepsy. These results suggest the development of novel methods of therapy, possibly including adjunctive antioxidant treatment. However, the study should be replicated in a larger sample of subjects.

\section{Ethical Considerations}

\section{Compliance with ethical guidelines}

This study was approved by the Medical Ethics Committee of Shahid Beheshti University of Medical Sciences (Core: IR.SBMU.RETECH.REC.1396.411). Written informed consent was obtained from all the participants.

\section{Funding}

This research did not receive any grant from funding agencies in the public, commercial, or non-profit sectors.

\section{Authors' contributions}

Conceptualization, investigation, and writing - original draft: Leila Simani and Omidvar Rezaei; Data collection: Fari Ryan, Masoumeh Sadeghi, Etrat Hooshmandi, and Mahtab Ramezani; Data analysis, Writing-review \& editing: LeilaSimani, Fari Ryan, Masoumeh Sadeghi, and Hossin Pakdaman.

\section{Conflict of interest}

The authors declared no conflict of interest.

\section{Acknowledgments}

We thank dr. Marjan Asadollahi, Mrs. Zohreh Afshar, and the Clinical Research Development Unit (CRDU) of Loghman Hakim Hospital and Shahid Beheshti University of Medical Sciences, Tehran, Iran for their support, cooperation, and assistance throughout the study period. 


\section{References}

Barros, D. O., Xavier, S. M. L., Barbosa, C. O., Silva, R. F., Freitas, R. L. M., \& Maia, F. D., et al. (2007). Effects of the vitamin E in catalase activities in hippocampus after status epilepticus induced by pilocarpine in Wistar rats. Neuroscience Letters, 416(3), 227-30. [DOI:10.1016/j.neulet.2007.01.057] [PMID]

Bhardwaj, M., \& Kumar, A. (2016). Neuroprotective mechanism of Coenzyme Q10 (CoQ10) against PTZ induced kindling and associated cognitive dysfunction: Possible role of microglia inhibition. Pharmacological Reports, 68(6), 1301-11. [DOI:10.1016/j.pharep.2016.07.005] [PMID]

Cengiz, M., Yüksel, A., \& Seven, M. (2000). The effects of carbamazepine and valproic acid on the erythrocyte glutathione, glutathione peroxidase, superoxide dismutase and serum lipid peroxidation in epileptic children. Pharmacological Research, 41(4), 423-5. [DOI:10.1006/phrs.1999.0603] [PMID]

Chew, G. T., \& Watts, G. F. (2004). Coenzyme Q10 and diabetic endotheliopathy: Oxidative stress and the 'recoupling hypothesis'. QJM: An International Journal of Medicine, 97(8), 537-48. [DOI:10.1093/qjmed/hch089] [PMID]

de Sales Santos, Í. M., de Freitas, R. L. M., da Silva, E. P., Feitosa C. M., Saldanha, G. B., \& Souza, G. F., et al. (2010). Effects of ubiquinone on hydroperoxide concentration and antioxidant enzymatic activities in the rat hippocampus during pilocarpine-induced seizures. Brain Research, 1315, 33-40. [DOI:10.1016/j.brainres.2009.12.037] [PMID]

El-ghoroury, E. A., Raslan, H. M., Badawy, E. A., El-Saaid, G. S., Agybi, M. H., \& Siam, I., et al. (2009). Malondialdehyde and coenzyme Q10 in platelets and serum in type 2 diabetes mellitus: Correlation with glycemic control. Blood Coagulation $\mathcal{E}$ Fibrinolysis, 20(4), 248-51. [DOI:10.1097/ MBC.0b013e3283254549] [PMID]

Ercegovac, M., Jović, N., Simić, T., Beslać-Bumbaširević, L., Sokić, D., \& Savić-Radojević, A., et al. (2013). Antiepileptic drugs affect protein, lipid and DNA oxidative damage and antioxidant defense in patients with epilepsy. Journal of Medical Biochemistry, 32(2), 121-30. [DOI:10.2478/jomb-2013-0009]

Gupta, Y., Briyal, S., \& Chaudhary, G. (2002). Protective effect of trans-resveratrol against kainic acid-induced seizures and oxidative stress in rats. Pharmacology Biochemistry and Behavior, 71(1-2), 245-9. [DOI:10.1016/S0091-3057(01)00663-3]

Lalkovičová, M., \& Danielisová, V. (2016). Neuroprotection and antioxidants. Neural Regeneration Research, 11(6), 865-74 [DOI:10.4103/1673-5374.184447] [PMID] [PMCID]

Lamperti, C., Naini, A., Hirano, M., De Vivo, D., Bertini, E. \& Servidei, S., et al. (2003). Cerebellar ataxia and coenzyme Q10 deficiency. Neurology, 60(7), 1206-8. [DOI:10.1212/01. WNL.0000055089.39373.FC] [PMID]

Martinc, B., Grabnar, I., \& Vovk, T. (2012). The role of reactive species in epileptogenesis and influence of antiepileptic drug therapy on oxidative stress. Current Neuropharmacology, 10(4), 328-43. [DOI:10.2174/157015912804499447] [PMID] [PMCID]

Martinc, B., Grabnar, I., \& Vovk, T. (2014). Antioxidants as a preventive treatment for epileptic process: A review of the current status. Current Neuropharmacology, 12(6), 527-50. [DO I:10.2174/1570159X12666140923205715] [PMID] [PMCID]
Matthews, R. T., Yang, L., Browne, S., Baik, M., \& Beal, M. F. (1998) Coenzyme Q10 administration increases brain mitochondrial concentrations and exerts neuroprotective effects. Proceedings of the National Academy of Sciences of the United States of America, 95(15), 8892-7. [DOI:10.1073/pnas.95.15.8892] [PMID] [PMCID]

Méndez-Armenta, M., Nava-Ruíz, C., Juárez-Rebollar, D., Rodríguez-Martínez, E., \& Yescas Gómez, P. (2014). Oxidative stress associated with neuronal apoptosis in experimental models of epilepsy. Oxidative Medicine and Cellular Longevity, 2014, 293689. [DOI:10.1155/2014/293689] [PMID] [PMCID]

Milanlioglu, A., Aslan, M., Ozkol, H., Çilingir, V., Aydın, M. N., \& Karadas, S. (2016). Serum antioxidant enzymes activities and oxidative stress levels in patients with acute ischemic stroke: Influence on neurological status and outcome. Wiener klinische Wochenschrift, 128(5-6), 169-74. [DOI:10.1007/s00508-015-0742-6] [PMID]

Militão, G. C. G., Ferreira, P. M. P., \& de Freitas, R. M. (2010). Effects of lipoic acid on oxidative stress in rat striatum after pilocarpine-induced seizures. Neurochemistry International, 56(1), 16-20. [DOI:10.1016/j.neuint.2009.08.009] [PMID]

Mancuso, M., Orsucci, D., Volpi, L., Calsolaro, V., \& Siciliano, G. (2010). Coenzyme Q10 in neuromuscular and neurodegenerative disorders. Current Drug Targets, 11(1), 111-21. https://www.ingentaconnect.com/content/ben/cdt/2010/00000011/00000001/ $\operatorname{art00014}$

Mohanan, P., \& Yamamoto, H. A. (2002). Preventive effect of melatonin against brain mitochondria DNA damage, lipid peroxidation and seizures induced by kainic acid. Toxicology Letters, 129(12), 99-105. [DOI:10.1016/S0378-4274(01)00475-1]

Ramezani, M., Sahraei, Z., Simani, L., Heydari, K., \& Shahidi, F. (2020). Coenzyme Q10 supplementation in acute ischemic stroke: Is it beneficial in short-term administration? Nutritional Neuroscience, 23(8), 640-5. [DOI:10.1080/1028415X.2018.1541269] [PMID]

Santos, Í. M. S., da Rocha Tomé, A., Saldanha, G. B., Ferreira, P. M. P. Militão, G. C. G., \& de Freitas, R. M. (2009). Oxidative stress in the hippocampus during experimental seizures can be ameliorated with the antioxidant ascorbic acid. Oxidative Medicine and Cellular Longevity, 2(4), 757814. [DOI:10.4161/oxim.2.4.8876] [PMID] [PMCID]

Sattarinezhad, E., Shafaroodi, H., Sheikhnouri, K., Mousavi, Z., \& Moezi, L. (2014). The effects of coenzyme Q10 on seizures in mice: The involvement of nitric oxide. Epilepsy \& Behavior, 37, 36-42. [DOI:10.1016/j.yebeh.2014.05.024] [PMID]

Simani, L., Ryan, F., Hashemifard, Sh., Hooshmandi, E., Madahi, M. \& Sahraei, Z., et al. (2018). Serum coenzyme Q10 is associated with clinical neurological outcomes in acute stroke patients. Journal of Molecular Neuroscience, 66(1), 53-8. [DOI:10.1007/s12031-018-11151] [PMID]

Spindler, M., Beal, M. F., \& Henchcliffe, C. (2009). Coenzyme Q10 effects in neurodegenerative disease. Neuropsychiatric Disease and Treatment, 5, 597-610. [DOI:10.2147/NDT.S5212] [PMID] [PMCID]

Sudha, K., Rao, A. V., \& Rao, A. (2001). Oxidative stress and antioxidants in epilepsy. Clinica Chimica Acta, 303(1-2), 19-24. [DOI:10.1016/S0009-8981(00)00337-5]

Tan, D. X., Manchester, L. C., Reiter, R. J., Qi, W., Kim, S. J., \& ElSokkary, G. H. (1998). Melatonin protects hippocampal neurons in vivo against kainic acid-induced damage in mice. Journal of Neuroscience Research, 54(3), 382-9. [DOI:10.1002/(SICI)10974547(19981101)54:3<382::AID-JNR9>3.0.CO;2-Y] [PMID] 
Tawfik, M. K. (2011). Coenzyme Q10 enhances the anticonvulsant effect of phenytoin in pilocarpine-induced seizures in rats and ameliorates phenytoin-induced cognitive impairment and oxidative stress. Epilepsy \& Behavior, 22(4), 671-7. [DOI:10.1016/j. yebeh.2011.09.018] [PMID]

Verrotti, A., Basciani, F., Trotta, D., Pomilio, M., Morgese, G., \& Chiarelli, F. (2002). Serum copper, zinc, selenium, glutathione peroxidase and superoxide dismutase levels in epileptic children before and after 1 year of sodium valproate and carbamazepine therapy. Epilepsy Research, 48(1-2), 71-5. [DOI:10.1016/S09201211(01)00322-9]

Xavier, S., Barbosa, C., Barros, D., Silva, R., Oliveira, A., \& Freitas, R. (2007). Vitamin C antioxidant effects in hippocampus of adult Wistar rats after seizures and status epilepticus induced by pilocarpine. Neuroscience Letters, 420(1), 76-9. [DOI:10.1016/j.neulet.2007.04.056] [PMID]

Yiş, U., Seçkin, E., Kurul, S. H., Kuralay, F., \& Dirik, E. (2009). Effects of epilepsy and valproic acid on oxidant status in children with idiopathic epilepsy. Epilepsy Research, 84(2-3), 232-7. [DOI:10.1016/j. eplepsyres.2009.02.003] [PMID] 
This Page Intentionally Left Blank 